\title{
THEORETICAL INVESTIGATION ON MULTIBUNCH INSTABILITIES IN ELECTRON STORAGE RINGS AND LINEAR ACCELERATORS
}

\author{
J. Gao, Laboratoire de L'Accélérateur Linéaire, IN2P3-CNRS \\ et Université de Paris-Sud, 91405 Orsay cedex, France
}

\section{Abstract}

In this paper we establish simple analytical criteria for the loaded quality factors of the dipole modes in the accelerating rf structures to prevent the multibunch instabilities in electron storage rings and linear accelerators.

\section{INTRODUCTION}

In the modern circular and future linear colliders, the option of multibunch operation mode has to be adopted to guarantee the required luminosity. Due to the long range transverse wake potentials, the transverse motion of a bunch in a bunch train can be influenced by the precedent bunches. If the long range transverse wake potentials are not properly controlled multibunch instabilities can occur and the luminosity will be degraded. The classical treatment of the multibunch instabilities can be found in ref. 1 for example. In this paper we try to treat the problem in a different way. We assume that each bunch is represented by a point charge and the detailed discussion about the single bunch longitudinal and transversal instabilities in electron storage rings can be found in refs. 2 and 3 .

A charged particle executes betatron oscillation in circular and linear accelerators can be regarded as an independent damped linear oscillator if there is no long range transverse wake potentials permitting the particle "talking" to its neighbours. The mechanisms of damping come from the synchrotron radiation in electron storage rings and the adiabatic acceleration in linear accelerators, respectively. The quality factor of this oscillator is related to the damping time and the betatron oscillation frequency. When the long range wake potentials are strong enough the particles in the bunch train will begin to be coupled from one to another and the independent oscillators become a chain of coupled oscillators with losses, and the betatron oscillation energies of the particles upstream can be transmitted to those of the particles downstream, known as multibunch instabilities. The physical picture described above is similar to that of a coupled rf cavity chain. Now, let's look at a chain of coupled rf cavities with losses which has been studied in detail in ref. 4 , one finds that to prevent the coupling between cavities the criterion $K_{c} Q<2$ should be satisfied, where $K_{c}$ is the coupling coefficient in the dispersion curve and $Q$ is the quality factor of the corresponding mode. By analogy, one can find the criteria under which the multibunch instabilities can be prevented in storage rings and linear accelerators.

\section{MULTIBUNCH INSTABILITIES IN ELECTRON STORAGE RINGS}

Particles in a storage ring execute betatron oscillations. If we neglect the effect of synchrotron radiation excitation and the long range wake potentials, the betatron motion of each bunch can be simplified as a damped oscillator expressed as

$$
y=A \cos \left(\omega_{y} \frac{s}{c}\right) \exp \left(-\frac{\omega_{y}}{2 Q_{y, r}}\left(\frac{s}{c}\right)\right)
$$

where $y$ denotes the transverse deviation in horizontal plane $x$ or vertical plane $z$ from the design orbit, $\omega_{y}$ is the angular betatron frequency, and $Q_{y, r}$ (the subscript $r$ denotes the storage ring case) is the quality factor of the oscillator expressed

$$
Q_{y, r}=\frac{\omega_{y} E_{0}}{<\mathcal{P}_{0}>J_{y}}
$$

where $\left\langle\mathcal{P}_{0}\right\rangle$ is the average synchrotron radiation power for one turn, $E_{0}$ is the particle energy, $J_{y}$ is the radiation damping partition number with $J_{y=x}=1-\mathcal{D}$ and $J_{y=z}=1(-2<\mathcal{D}<1)$. In reality, however, charged particles interact with the environment and produce long range wake potentials which make the independent oscillating oscillator become a coupled oscillator chain. The coupling coefficient $K_{c, r}$ between the two successive bunches can be calculated from the coherent frequency change due to the long range wake potential similar to the single bunch case [3]

$$
\left|\frac{\Delta \nu_{y, c}}{\nu_{y}}\right|=\frac{e^{2} N_{e} W_{\perp}^{\prime}\left(s_{b}\right) \overline{\beta_{y, c}}}{\nu_{y} E_{0}}
$$

where $W_{\perp}^{\prime}\left(s_{b}\right)(\mathrm{V} / \mathrm{C} / \mathrm{m})$ is the long range dipole wake potential of one turn and of unit transverse displacement, $s_{b}$ is the distance between two successive bunches, $N_{e}$ is the particle population in the bunch, $\overline{\beta_{y, c}}$ is the average beta function at the position of the rf cavities, and $\nu_{y}$ is the tune number. By analogy with a coupled rf cavity chain, one finds the coupling coefficient expressed as follows

$$
K_{c, r}=2\left|\frac{\Delta \nu_{y, c}}{\nu_{y}}\right|
$$

According ref. 4, one knows that under the condition

$$
K_{c, r} Q_{y, r}<2
$$

there will be no coupling between two successive oscillators. From eqs. 2 and 4, one finds

$$
W_{\perp}^{\prime}<\frac{<\mathcal{P}_{0}>J_{y}}{2 \pi f_{0} e^{2} N_{e} \overline{\beta_{y, c}}}
$$




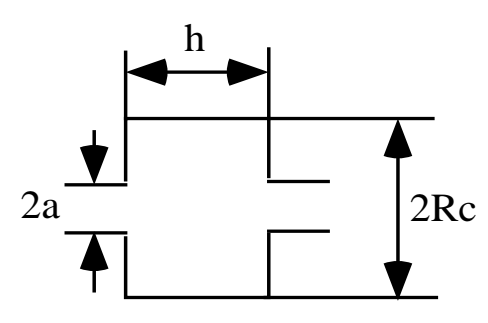

Figure 1: A single rf cavity.

where $f_{0}$ is the revolution frequency. For an isomagnetic ring

$$
W_{\perp}^{\prime}<\frac{\gamma^{4} J_{y}}{6 \pi \epsilon_{0} \rho N_{e} \overline{\beta_{y, c}}}
$$

where $\rho$ is the local bending radius and $\gamma$ is the normalized particle energy. In a storage ring the accelerating rf cavities are the main components which produce long range wake potentials (narrow band impedance). In the following one considers only the $\mathrm{TM}_{110}$ mode in the accelerating rf cavities since $W_{\perp}^{\prime}\left(s_{b}\right) \approx W_{\perp, 110}^{\prime}\left(s_{b}\right)$ for the long range wake potential. The $\mathrm{TM}_{110}$ mode wake potential can be expressed as

$$
\begin{gathered}
W_{\perp, 110}^{\prime}=N_{c} h \frac{2 c K_{1}}{\omega_{r f, 1} a^{2}} \sin \left(\omega_{r f, 1} \frac{s_{b}}{c}\right) \times \\
\exp \left(-\frac{\omega_{r f, 1}}{2 Q_{1, r}}\left(\frac{s_{b}}{c}\right)\right) \exp \left(-\frac{\omega_{r f, 1}^{2} \sigma_{z}^{2}}{2 c^{2}}\right)
\end{gathered}
$$

where $N_{c}$ is the number of the cavities in the ring, $h$ is the inner length of the cavity, $\sigma_{z}$ is the rms bunch length ( $\sigma_{z}$ is used to calculate the transverse wake potential and the point charge assumption is still valid), $\omega_{r f, 1}$ and $Q_{1, r}$ are the angular frequency and the loaded quality factor of the dipole mode, respectively. According to ref. 5, $K_{1}$ in eq. 8 corresponding to a single cavity can be expressed analytically as follows

$$
\begin{gathered}
K_{1}=\frac{J_{1}^{2}\left(\frac{u_{11}}{R_{c}} a\right)}{\epsilon_{0} \pi R_{c}^{2} J_{2}^{2}\left(u_{11}\right)} S\left(x_{1}\right)^{2} \\
S(x)=\frac{\sin x}{x} \\
x_{1}=\frac{h u_{11}}{2 R_{c}} \\
\omega_{r f, 1} \approx \frac{c u_{11}}{R_{c}}
\end{gathered}
$$

where $R_{c}$ is the cavity radius, $a$ is the iris radius as shown in Fig. 1, and $u_{11}=3.832$ is the first root of the first order Bessel function. $\omega_{r f, 1}$ in eq. 12 can be rather precisely determined by using the analytical formulae from perturbation methods [6][7]. Being pessimistic, we assume $\sin \left(\omega_{r f, 1} \frac{s_{b}}{c}\right)=1$ and find consequently from eq. 7 that

$$
\exp \left(-\frac{\omega_{r f, 1}}{2 Q_{1, r}}\left(\frac{s_{b}}{c}\right)\right)<
$$

$$
\frac{\gamma^{4} \omega_{r f, 1} a^{2} J_{y}}{12 \pi c \epsilon_{0} \rho N_{c} h N_{e} K_{1} \overline{\beta_{y, c}} \exp \left(-\frac{\omega_{r f, 1}^{2} \sigma_{z}^{2}}{2 c^{2}}\right)}
$$

and

$$
Q_{1, r}<\frac{u_{11} s_{b}}{2 R_{c} \ln \left(\frac{12 \pi \epsilon_{0} \rho R_{c} N_{c} h N_{e} K_{1} \overline{\beta_{y, c}} \exp \left(-\frac{u_{11}^{2} \sigma_{z}^{2}}{2 R^{2}}\right)}{\gamma^{4} u_{11} a^{2} J_{y}}\right)}
$$

To reach the required loaded $Q_{1, r}$, waveguide type higher order mode couplers can be installed on the accelerating rf cavities and the dimensions of the coupling apertures can be determined analytically as shown in ref. 8. From eq. 14 one can find the condition under which the dipole mode need not to be damped. This condition is simply that $Q_{1, r} \rightarrow \infty$ (this condition is somewhat strong but very useful since it doesn't depend on the specific unloaded dipole mode quality factor) when $N_{e}$ satisfies

$$
N_{e} \leq N_{e}^{*}=\frac{\gamma^{4} u_{11} a^{2} J_{y}}{12 \pi \epsilon_{0} \rho R_{c} N_{c} h K_{1} \overline{\beta_{y, c}} \exp \left(-\frac{u_{11}^{2} \sigma_{z}^{2}}{2 R^{2}}\right)}
$$

Taking Beijing Tau-Charm Factory (BTCF) parameters for example, from eq. 14 one gets $Q_{1, r}=99$ with $s_{b}=12$ $\mathrm{m}, R_{c}=0.224 \mathrm{~m}, h=0.22 \mathrm{~m}, a=0.044 \mathrm{~m}, K_{1}=$ $1.4 \times 10^{11}(\mathrm{~V} / \mathrm{C} / \mathrm{m}), N_{c}=12, N_{e}=1.5 \times 10^{11}, \overline{\beta_{y, c}}=10$ $\mathrm{m}, \sigma_{z}=0.01 \mathrm{~m}, J_{y=z}=1, \rho=8.58 \mathrm{~m}$, and $E_{0}=2 \mathrm{GeV}$. This result justifies what has been found in ref. 10 .

\section{MULTIBUNCH INSTABILITIES IN LINEAR ACCELERATORS}

In a linear accelerator the physical picture is a little bit different from that in a storage ring. The betatron motion can still be written as that in eq. 1, the quality factor, however, is expressed as

$$
Q_{y, L}=\frac{\omega_{y} E_{0}}{c e E_{z}}
$$

where $E_{z}$ is the accelerating gradient and the subscript $L$ in this section denotes linear accelerator case. The damping effect is due to the fact that a particle is accelerated continuously and the betatron oscillation is adiabatically damped [9]. The relative coherent betatron oscillation frequency variation due to the long range transverse wakefield is

$$
\left|\frac{\Delta \omega_{y, c}}{\omega_{y}}\right|=\frac{2 \pi e^{2} N_{e} c W_{\perp, L}^{\prime}\left(s_{b}\right) \overline{\beta_{y}}}{\omega_{y} E_{0}}
$$

where $W_{\perp, L}^{\prime}\left(s_{b}\right)\left(\mathrm{V} / \mathrm{C} / \mathrm{m}^{2}\right)$ is the long range transverse wakefield strength of unit transverse displacement and $\overline{\beta_{y}}$ is the average beta function value in the linac. In the following one considers only the $\mathrm{TM}_{110}$ mode in the accelerating rf structures since $W_{\perp, L}^{\prime}\left(s_{b}\right) \approx W_{\perp, L, 1}^{\prime}\left(s_{b}\right)$ for the long range wakefield, where $W_{\perp, L, 110}^{\prime}\left(s_{b}\right)$ is the $\mathrm{TM}_{110}$ wakefield expressed as

$$
W_{\perp, L, 110}^{\prime}=\frac{2 c K_{1, L}}{\omega_{r f, 1, L} a^{2}} \sin \left(\omega_{r f, 1, L} \frac{s_{b}}{c}\right) \times
$$




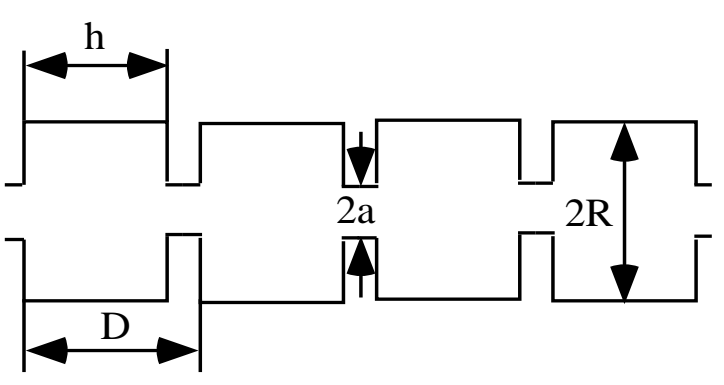

Figure 2: A disk-loaded accelerating structure.

$$
\exp \left(-\frac{\omega_{r f, 1, L}}{2 Q_{1, L}}\left(\frac{s_{b}}{c}\right)\right) \exp \left(-\frac{\omega_{r f, 1, L}^{2} \sigma_{z}^{2}}{2 c^{2}}\right) F\left(s_{b}\right)
$$

where $\omega_{r f, 1, L}$ and $Q_{1, L}$ are the synchronous frequency and the loaded quality factor of the $\mathrm{TM}_{110}$ mode passband, respectively, and $F(s)$ is the wakefield reduction function comes from the detuning effect (for a constant impedance accelerating structure $F \equiv 1$ ). According to ref. 5, one knows that

$$
\begin{gathered}
K_{1, L}=\frac{h J_{1}^{2}\left(\frac{u_{11}}{R} a\right)}{\epsilon_{0} \pi D R^{2} J_{2}^{2}\left(u_{11}\right)} S\left(x_{1, L}\right)^{2} \\
x_{1, L}=\frac{h u_{11}}{2 R} \\
\omega_{r f, 1, L} \approx \frac{c u_{11}}{R}
\end{gathered}
$$

where $D$ is the period length of the accelerating structure, $h$ is the inner cavity length, $a$ and $R$ are the iris and the cavity radius, respectively, as shown in Fig. 2. Knowing

$$
K_{c, L}=2\left|\frac{\Delta \omega_{y, c}}{\omega_{y}}\right|
$$

and letting $Q_{y, L} K_{c, L}<2$, one finds

$$
W_{\perp, L, 1}^{\prime}<\frac{E_{z}}{2 \pi e N_{e} \overline{\beta_{y}}}
$$

Taking $\sin \left(\omega_{r f, 1, L} \frac{s_{b}}{c}\right)=1$ for the pessimistic case, one finds

$$
\begin{gathered}
\exp \left(-\frac{\omega_{r f, 1, L}}{2 Q_{1, L}}\left(\frac{s_{b}}{c}\right)\right)< \\
\frac{E_{z} \omega_{r f, 1, L} a^{2}}{4 \pi c e N_{e} R K_{1, L} \overline{\beta_{y}} \exp \left(-\frac{\omega_{r f, 1, L}^{2} \sigma_{z}^{2}}{2 c^{2}}\right) F\left(s_{b}\right)}
\end{gathered}
$$

and

$$
Q_{1, L}<\frac{u_{11} s_{b}}{2 R \ln \left(\frac{4 \pi e N_{e} R K_{1, L} \overline{\beta_{y}} \exp \left(-\frac{u_{11}^{2} \sigma_{z}^{2}}{2 R^{2}}\right) F\left(s_{b}\right)}{E_{z} u_{11} a^{2}}\right)}
$$

Similar to the storage ring case, one gets the condition under which no higher order mode coupler is needed

$$
N_{e} \leq N_{e}^{*}=\frac{E_{z} u_{11} a^{2}}{4 \pi e R K_{1, L} \overline{\beta_{y}} \exp \left(-\frac{u_{11}^{2} \sigma_{z}^{2}}{2 R^{2}}\right) F\left(s_{b}\right)}
$$

Here we give an example of an ideal detuned S-band linear accelerating structure. From eq. 25 one finds $Q_{1, L}=2740$ with $s_{b}=5 \mathrm{~m}, R=0.04 \mathrm{~m}, h=0.0292$ $\mathrm{m}, D=0.035 \mathrm{~m}, a=0.01 \mathrm{~m}, K_{1, L}=10 \times 10^{12}(\mathrm{~V} / \mathrm{C} / \mathrm{m})$, $N_{e}=2 \times 10^{10}, \overline{\beta_{y}}=85 \mathrm{~m}, \sigma_{z}=0.005 \mathrm{~m}, F\left(s_{b}\right)=0.0065$, and $E_{z}=17 \mathrm{MV} / \mathrm{m}$. If a constant impedance structure is used then $Q_{1, L}=187$ for the the same set of parameters.

\section{CONCLUSION}

In this paper we give simple criteria to determine the loaded quality factors of the dipole modes in the accelerating $\mathrm{rf}$ structures which are responsible for the multibunch instabilities in electron storage rings and linear accelerators. The relation between the beam and the machine parameters are well established, and the analytical criteria can be served as scaling laws to optimize the machine performance.

\section{REFERENCES}

[1] A. Chao, "Physics of collective beam instabilities in high energy accelerators", John Wiley \& Sons, Inc. 1993.

[2] J. Gao, "Bunch lengthening and energy spread increasing in electron storage rings", these Proceedings.

[3] J. Gao, "Theory of single bunch transverse collective instabilities in electron storage rings", these Proceedings.

[4] J. Gao, "The criterion for the coupling states between cavities with losses", Nucl. Instr. and Meth. A325 (1995) 661662.

[5] J. Gao, "Analytical formulae and the scaling laws for the loss factors and the wakefields in disk-loaded periodic structures", Nucl. Instr. and Meth. A381 (1996) 174-177.

[6] J. Gao, "Analytical formulae for the frequency changes due to openning apertures on cavity walls", Nucl. Instr. and Meth. A311 (1992) 437.

[7] J. Gao, "Analytical approach and scaling laws in the design of disk-loaded travelling wave accelerating structures", Particle Accelerators, Vol. 43(4) (1994) pp. 235-257.

[8] J. Gao, "Analytical researches on the accelerating structures, wakefields, and beam dynamics for future linear colliders", LAL 96-45, May 1996, memoir for the habilitation to direct researches, Université de Paris-Sud, Centre d'Orsay, p. 90.

[9] H. Wiedemann, "Particle Accelerator Physics I", SpringerVerlag, 1993, p. 61.

[10] BTCF group, "Feasibility study report on Beijing TauCharm Factory”, IHEP-BTCF report 01, December 1995. 